\title{
PYODERMA GANGRENOSUM AS THE FIRST MANIFESTATION OF SYSTEMIC LUPUS ERYTHEMATOSUS: A CASE REPORT
}

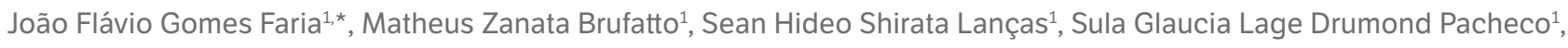
Andrea de Almeida Peduti Batista ${ }^{1}$, Laura Maria Silva de Siqueira ${ }^{1}$, Douglas Squizatto Leite ${ }^{1}$, Daniela Esteves Temporim ${ }^{1}$, Henrique Pereira Sampaio ${ }^{1}$, Luiz Eduardo Valente ${ }^{1}$
\end{abstract}

1.Faculdade de Medicina de Botucatu, Botucatu (SP), Brazil.

*Corresponding author: joao_flavio_faria@hotmail.com

\section{BACKGROUND}

Pyoderma gangrenosum (PG) is a rare neutrophilic dermatosis that, in some situations, can be the manifestation of a systemic disease. There are reports in the literature of isolated cases of PG and rheumatic diseases, especially rheumatoid arthritis. We describe the case of a patient who presented PG as the initial manifestation of systemic lupus erythematosus (SLE).

\section{CASE REPORT}

A 21-year-old female patient was admitted to our service with a 6-month history of deep and bleeding ulcers in different regions of the body. On physical examination, she presented a 4-cm deep ulcerated lesion in the genital region, a 2-cm deep ulcer in the left inguinal region, a 3-cm bleeding ulcer in the wrist region, and a 1.5-cm deep ulcer in the palate. She also had livedo reticularis, oligoarticular arthritis, and macroscopic hematuria. From personal history, she mentioned pre-eclampsia in a previous pregnancy, evolving with systemic arterial hypertension. In laboratory tests, she presented hematuria, mild proteinuria, creatinine =2.9, microcytic hypochromic anemia, leukopenia by lymphopenia, mild thrombocytopenia, ESR $72 \mathrm{~mm} / \mathrm{h}$, direct Coombs positive, and no clotting disorder. Diagnostic hypotheses of SLE, antiphospholipid syndrome, vasculitis-Behçet or granulomatosis with polyangiitis, infectious contagious differentials that could course with ulcers (acute HIV, syphilis, leishmaniasis) and lymphoproliferative diseases were raised. Therefore, our team opted for the introduction of antibiotic therapy with amoxicillin and clavulanate, prednisone $0.5 \mathrm{mg} / \mathrm{kg}$. The biopsy of the ulcer showed that it was compatible with pyoderma gangrenosum. Renal biopsy (glomerulonephritis by mesangial proliferative immune complexes) and bone marrow biopsy (without infiltration by infectious or neoplastic agents) were performed. In the evolution, the patient presented an immune panel with homogeneous nuclear ANA 1/160, high positive anti-DNA, consumption of $\mathrm{C} 3$ and $\mathrm{C} 4$, high positive $\lg \mathrm{G}$ and $\lg \mathrm{M}$ anticardiolipins, being opted for therapy with methylprednisolone $1 \mathrm{~g} /$ day for 3 days followed by cyclophosphamide $0.6 \mathrm{~g} / \mathrm{m}^{2}$. The patient had progressive improvement, being discharged from the hospital using Prednisone $1 \mathrm{mg} / \mathrm{kg}$ and maintaining a monthly therapy schedule with cyclophosphamide for 6 months. After completion of therapy programming, the patient was asymptomatic with significant improvement in renal function and no recurrence of pyoderma gangrenosum.

\section{CONCLUSION}

Although the association of PG with SLE is rare, assistant doctors should be aware of important cutaneous manifestations and their association with systemic diseases. Systemic lupus erythematosus presents an important diversity of symptoms, requiring the assistant team to search for appropriate differential diagnoses before the introduction of immunosuppressive treatment.

\section{KEYWORDS}

Pyoderma Gangrenosum, SLE, Nephritis. 